\title{
Testosterona e gonadotrofina coriônica humana estimulam a esteroidogênese em células da granulosa de folículo pré-ovulatório de égua?
}

\author{
[Do testosterone and human chorionic gonadotropin stimulate steroidogenesis in granulosa \\ cells of preovulatory follicle in mare?] \\ M.C. Caldas-Bussiere ${ }^{1}$, J.M. Garcia ${ }^{2}$, J.C. Barbosa ${ }^{2}$, A.A.M. Rosa e Silva ${ }^{3}$ \\ ${ }^{1}$ Centro de Ciências e Tecnologias Agropecuárias \\ Universidade Estadual do Norte Fluminense Darcy Ribeiro \\ Av. Alberto Lamego, 2000 - Parque Califórnia \\ 28013-602 - Campos dos Goytacazes, RJ \\ ${ }^{2}$ Faculdade de Ciências Agrárias e Veterinárias - UNESP - Jaboticabal, SP \\ ${ }^{3}$ Instituto de Ciências Fisiológicas - UNB - Brasília, DF
}

\begin{abstract}
RESUMO
Avaliou-se o papel da gonadotrofina coriônica humana (hCG) e da testosterona na produção de progesterona $\left(\mathrm{P}_{4}\right)$ e $17 \beta$-estradiol $\left(\mathrm{E}_{2}\right)$ pelas células da granulosa cultivadas in vitro de folículo antral de égua. Os tratamentos usados foram: 1- controle (nenhum hormônio adicionado), 2- $1 \mathrm{UI}$ hCG $(0,3 \mu \mathrm{g} / \mathrm{ml}) \mathrm{e}$ 3- 10UI hCG $(3,0 \mu \mathrm{g} / \mathrm{ml})$. O tratamento com hCG foi realizado na presença ou não de testosterona $(144 \mathrm{ng} / \mathrm{ml})$. O meio foi coletado e substituído com $0,25,3,6,12,24$ e $144 \mathrm{~h}$ de cultivo. As concentrações de $\mathrm{P}_{4}$ e $E_{2}$ foram mensuradas por radioimunoensaio. Não se observou diferença entre os tratamentos 1 e 3 quanto à produção de $\mathrm{P}_{4}$ e $\mathrm{E}_{2}$; o tratamento 1 resultou em aumento da concentração de progesterona após $24 \mathrm{~h}$ de cultura $(\mathrm{P}<0,01)$, mas somente em presença de testosterona. A concentração de estradiol aumentou em presença de testosterona, alcançando concentração máxima com $6 \mathrm{~h}$ de cultura $(\mathrm{P}<0,01)$, e diminuiu gradativamente, até atingir a concentração observada com $0,25 \mathrm{~h}$ de cultura. A adição de hCG não influenciou a síntese do estradiol. A testosterona desempenhou importante efeito estimulador na síntese/secreção doe $\mathrm{E}_{2}$ pelas células da granulosa e modulou a ação do hormônio luteinizante na diferenciação e luteinização das células da granulosa de folículo antral presumidamente pré-ovulatório de égua in vitro.
\end{abstract}

Palavras-chave: égua hormônio luteinizante, testosterona, esteroidogênese, células da granulosa

\begin{abstract}
The role of the human chorionic gonadotropin $(h C G)$ and testosterone was evaluated in the progesterone $\left(P_{4}\right)$ and estradiol-17 $\left(E_{2}\right)$ production by granulos a cells of antral follicles from mare cultivated in vitro. The treatment (groups) with gonadotropin consisted of: 1-control (no added hormone); $2-1$ IU hCG $(0.3 \mathrm{mg} / \mathrm{ml})$ and 3- $10 \mathrm{IU} \mathrm{hCG}(3.0 \mathrm{mg} / \mathrm{ml})$. The treatment with $\mathrm{hCG}$ was carried out in the presence or not of testosterone $(144 \mathrm{ng} / \mathrm{ml})$. The culture medium was collected and replaced at $0.25,3,6,12,24$ and $144 \mathrm{~h}$ of culture. The concentrations of $P_{4}$ and $E_{2}$ were measured by radioimunoassay. Analyses of variance were used for $P_{4}$ and $E_{2}$, and mean of the factors were compared by the Tukey test at $5 \%$ of probability. No difference was observed between 1 and 2 groups. Treatment with 1 IU of hCG increased progesterone concentration after $24 \mathrm{~h}$ of culture $(P<0.01)$, only in the presence of testosterone. The concentration of estradiol increased in the presence of testosterone, reaching maximum concentration with 6 h of culture $(P<0.01)$, and reduced gradually until the observed concentration at $0.25 \mathrm{~h}$ of culture. The addition of
\end{abstract}

Recebido para publicação em 14 de julho de 2003

Recebido para publicação, após modificações, em 16 de abril de 2004

E-mail: claracal@uenf.br 
$h C G$ had no effect in the synthesis of this steroid. The testosterone modulates the action of the luteinizing hormone in the differentiation and luteinization of the granulosa cells and plays important estimulator effect in the synthesis/secretion of $E_{2}$ by granulosa cells in vitro of presumptive pre-ovulatory antral follicle in mare.

Keywords: mare, luteinizing hormone, testosterone, steroidogenesis, granulosa cell

\section{INTRODUÇÃO}

As células da granulosa (CG) e as células da teca (CT) interagem para permitir a síntese de $\mathrm{E}_{2}$ (Falk, 1959). O modelo 2 células/2 hormônios para a produção de $\mathrm{E}_{2}$ em folículos antrais préovulatórios de suínos (Stoklosowa et al., 1982), bovinos (Fortune, 1986) e eqüinos (Sirois et al., 1991) demonstra que as CT interna são estimuladas pelo hormônio luteinizante (LH) e produzem andrógenos, enquanto que o hormônio folículo estimulante (FSH) (e $\mathrm{LH}$ em folículos maturados) estimula as CG para aromatizarem os andrógenos sintetizados pelas CT.

A testosterona $(\mathrm{T})$ estimula a síntese/liberação de progesterona $\left(\mathrm{P}_{4}\right)$ pelas $\mathrm{CG}$ em presença de $\mathrm{LH}$ em fêmeas de ratos (Liu e Hseuh, 1986), bovinos (Fortune, 1986) e suínos (Stoklosowa et al., 1982), contudo nenhum efeito da T na síntese de $\mathrm{P}_{4}$ ou $\mathrm{E}_{2}$ foi descrito em eqüinos.

O objetivo da presente investigação foi estudar o papel da testosterona e da gonadotrofina coriônica humana (hCG) na síntese de $\mathrm{P}_{4}$ e $\mathrm{E}_{2}$ pelas CG de folículo antral presumivelmente préovulatório de eqüino.

\section{MATERIAL E MÉTODOS}

Ovários de éguas cíclicas vazias foram obtidos do Frigorífico Pomar, Araguari, MG, e imediatamente colocados em frascos estéries contendo PBS, gentamicina $(50 \mu \mathrm{g} / \mathrm{ml})$ e anfotericina $B^{1} \quad(1 \mu \mathrm{g} / \mathrm{ml})$ na temperatura ambiente e transportados até o laboratório.

Um folículo ovariano contendo $55 \mathrm{ml}$ de fluido folicular foi selecionado de acordo com as características morfológicas (tamanho, coloração e intensidade de vascularização) após a dissecção do tecido ovariano no fluxo laminar com a ajuda

\footnotetext{
${ }^{1}$ Bristol-Myers Squibb, New York, NY, USA
}

de pinças e tesouras esterilizadas. Após a coleta do fluido folicular, o folículo foi seccionado, invertido e colocado em placa de Petri contendo PBS. As CG foram obtidas raspando-se delicadamente a face interna do folículo com uma pinça. Posteriormente, foram centrifugadas $(200 \mathrm{~g} / 10 \mathrm{~min})$ duas vezes em PBS e ressuspendidas em $3 \mathrm{ml}$ de meio de cultura, homogeinizadas com repetidas aspirações com pipeta Pasteur e contadas em câmara de Newbauer. A viabilidade das CG foi determinada utilizando-se a coloração vital "triplan-blue", sendo consideradas como vivas as células não coradas.

As CG foram cultivadas (200.000 células vivas/ml meio) em placas de 24 poços $^{2}$ em meio de cultivo de tecidos (TCM) $199^{3}$ suplementado com cortisol $^{3}(40 \mathrm{ng} / \mathrm{ml})$, insulina ${ }^{3}(1 \mu \mathrm{g} / \mathrm{ml})$, transferrina $^{3}(5 \mu \mathrm{g} / \mathrm{ml})$ e $10 \%$ de soro fetal bovino $^{4}$, de acordo com Sirois et al. (1990). Visto que o hCG em CG de éguas apresenta atividade biológica semelhante ao LH (Kelly et al., 1988), ele foi utilizado neste experimento para se avaliar o efeito do LH na esteroidogênese ovariana. $\mathrm{O}$ tratamento com $\mathrm{hCG}^{3}$ constou de: 1controle (nenhum hormônio adicionado); 2- 1UI hCG $(0,3 \mu \mathrm{g} / \mathrm{ml})$ e $3-10 \mathrm{UI}$ hCG $(3 \mu \mathrm{g} / \mathrm{ml})$. Cada tratamento com hCG foi realizado em triplicata com ou sem $\mathrm{T}^{3}(0.5 \mu \mathrm{M} ; 144 \mathrm{ng} / \mathrm{ml})$. O meio foi coletado após $0,25,3,6,12,24,48$ e $144 \mathrm{~h}$ de cultura e estocado a $-20^{\circ} \mathrm{C}$ até o dia do ensaio hormonal de $\mathrm{P}_{4}$ e $\mathrm{E}_{2}$. As culturas foram incubadas a $37^{\circ} \mathrm{C}$ em estufa com atmosfera umidificada com $95 \%$ de ar e $5 \%$ de $\mathrm{CO}_{2}$.

Utilizou-se um esquema fatorial $3 \times 2 \times 7$, três concentrações de hCG (zero, 1 e $10 \mathrm{UI}$ ), com e sem $\mathrm{T}$, e sete tempos de cultura, em delineamento inteiramente ao acaso, com três repetições. Os resultados referentes à produção

\footnotetext{
${ }^{2}$ Corning, Corning, NY, USA

${ }^{3}$ Sigma-Aldrich, St Louis, MO, USA

${ }^{4}$ Gibco BRL, Grand Island, NY, USA
} 
de $\mathrm{P}_{4}$ e $\mathrm{E}_{2}$ pelas $\mathrm{CG}$ cultivadas in vitro foram submetidos à análise de variância e as médias comparadas pelo teste de Tukey ao nível de 5\% de probabilidade (Gomes, 1987).

As concentrações de $\mathrm{P}_{4}$ (Rosa e Silva et al., 1993) e $E_{2}$ no meio de cultura (Bélanger et al., 1980) foram determinadas por radioimunoensaio. $\mathrm{O}$ coeficiente de variação (CV) inter e intra-ensaio para $\mathrm{P}_{4}$ foi de $10,3 \%$ e $5,0 \%$, respectivamente, e a dose mínima detectável foi de $6,25 \mathrm{pg} /$ tubo. O CV inter e intra-ensaio para $\mathrm{E}_{2}$ foi de $5,8 \%$ e $3,1 \%$, respectivamente, e a dose mínima detectável foi de $1,3 \mathrm{pg} /$ tubo.

\section{RESULTADOS}

A síntese de $\mathrm{P}_{4}$ pelas $\mathrm{CG}$ in vitro não foi afetada pela adição de 1 UI ou 10 UI de hCG no meio de cultura em ausência de $\mathrm{T}$ (Fig. 1). A $\mathrm{T}$ na ausência do hCG (controle) não alterou as concentrações de $\mathrm{P}_{4}$ no meio de cultura. Quando o meio de cultura foi suplementado com T (144 $\mathrm{ng} / \mathrm{ml})$ e $1 \mathrm{UI}$ de hCG, observou-se aumento de oito vezes (40 para $430 \mathrm{ng} / \mathrm{ml})$ na concentração de $\mathrm{P}_{4}$ no meio de cultura $(\mathrm{P}<0,01)$. Não houve efeito da $\mathrm{T}$ quando $10 \mathrm{UI}$ de $\mathrm{hCG}$ foram adicionadas (Fig. 1).

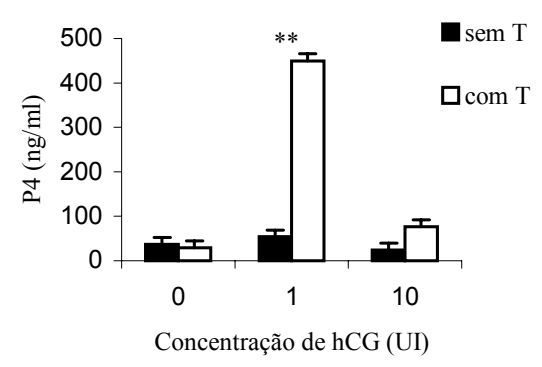

Figura 1. Concentração cumulativa (média \pm erro padrão da média) de progesterona $\left(\mathrm{P}_{4}\right)$ no meio de cultura de células da granulosa (CG) de folículo antral pré-ovulatório. As CG foram incubadas no meio TCM 199 na ausência (ם-口) ou na presença $(\square-\square)$ de testosterona $(\mathrm{T} ; 144 \mathrm{ng} / \mathrm{ml})$ e hCG $(0,1$ e $10 \mathrm{UI}$ hCG) por um período de $144 \mathrm{~h}(\mathrm{P}<0,01)$.
Concentração basal de $\mathrm{E}_{2}$ foi observada no meio de cultura (2,98ng/ml) na ausência de T (Fig. 2). $\mathrm{A}$ adição de $\mathrm{T}(144 \mathrm{ng} / \mathrm{ml})$ ao meio de cultura aumentou 506 vezes a concentração de $\mathrm{E}_{2}$ $(\mathrm{P}<0,01)$. $\mathrm{O}$ tratamento com hCG não alterou o acúmulo de $\mathrm{E}_{2}$ na cultura na presença ou ausência de T (Fig. 2).

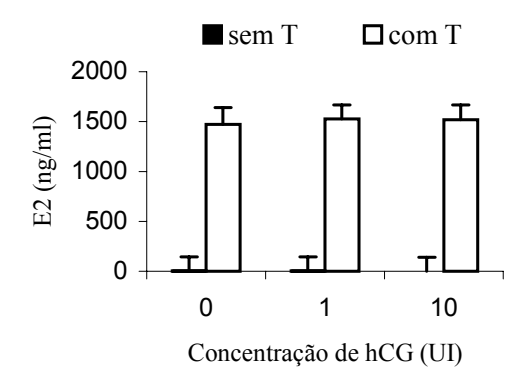

Figura 2. Concentração cumulativa (média \pm erro padrão da média) de $17 \beta$-estradiol $\left(\mathrm{E}_{2}\right)$ no meio de cultura de células da granulosa (CG) de folículo antral pré-ovulatório. As CG foram incubadas em meio TCM 199 na ausência ( $\square-\square)$ ou na presença $(\square-\square)$ de testosterona (T; 144ng/ml) e hCG $(0,1$ e 10 UI hCG) por um período de $144 \mathrm{~h}$.

A concentração de $\mathrm{P}_{4}$ no meio de cultura na presença de $\mathrm{T}$ e 1UI de hCG aumentou significativamente $(\mathrm{P}<0,01)$ com $24 \mathrm{~h}$ de cultura, atingindo concentração máxima após 144h (Fig. 3). Concentração basal foi observada quando $0 \mathrm{e}$ 10UI de hCG foram adicionados ao meio de cultura. Já a concentração de $\mathrm{E}_{2}$ no meio de cultura na presença de $\mathrm{T}$ foi máxima após $6 \mathrm{~h}$ de cultura $(\mathrm{P}<0,01)$, diminuindo gradativamente após esse período. A adição de hCG ao meio de cultura não alterou a síntese/secreção de $\mathrm{E}_{2}$ pelas CG durante o período de cultura (Fig. 4). Relação temporal inversa foi observada entre a concentração de $\mathrm{P}_{4}$ e de $\mathrm{E}_{2}$ no meio de cultura ao longo das $144 \mathrm{~h}$ quando foi adicionado $1 \mathrm{UI}$ de hCG em presença da T (Fig. 3 e 4). 


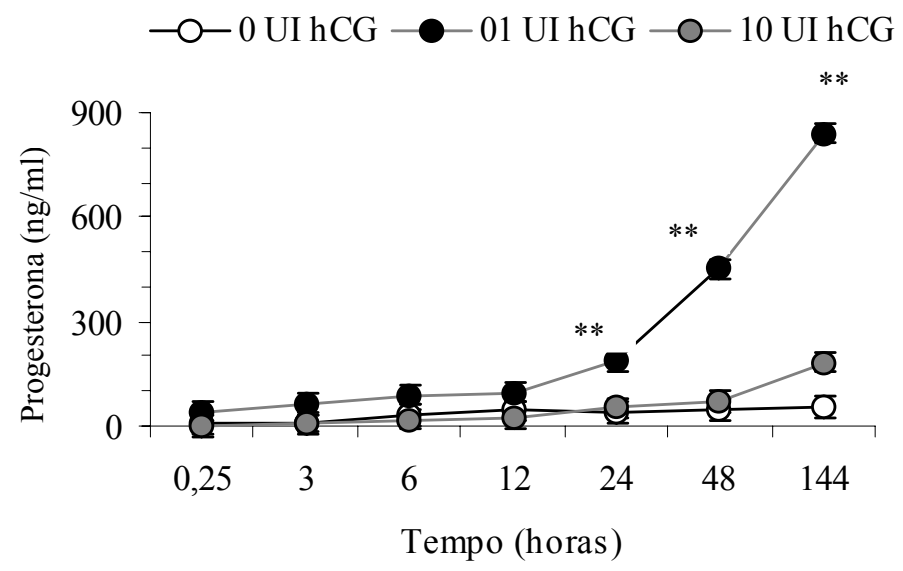

Figura 3. Concentração de progesterona no meio de cultura de células da granulosa (CG) de folículo antral pré-ovulatório durante $0,25,3,6,12,24,48$ e 144h. As CG foram incubadas em meio TCM 199 suplementado com testosterona $(144 \mathrm{ng} / \mathrm{ml})$ e $0 \mathrm{UI}\left(\mathrm{o}_{-} \mathrm{o}\right)$, $1 \mathrm{UI}(\bullet \bullet)$ e $10 \mathrm{UI}(\bullet \bullet)$ de $\mathrm{hCG}$. Média \pm EPM $(\mathrm{P}<0,01)$.

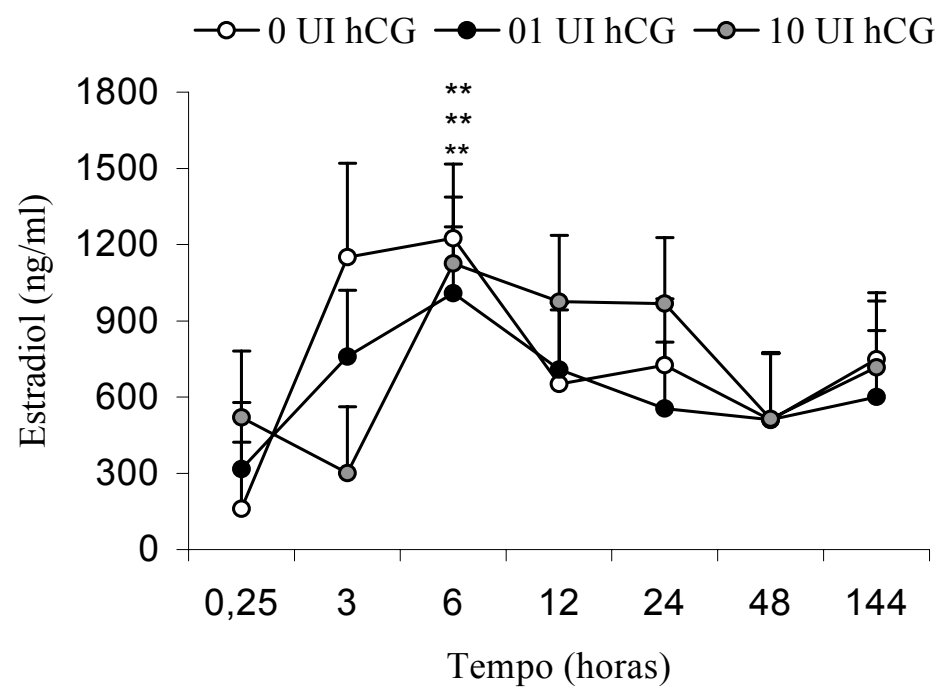

Figura 4. Concentração de $17 \beta$-estradiol $\left(\mathrm{E}_{2}\right)$ no meio de cultura de células da granulosa $(\mathrm{CG})$ de folículo antral pré-ovulatório durante $0,25,3,6,12,24,48$ e 144h. As CG foram incubadas em meio TCM 199 suplementado com testosterona $(144 \mathrm{ng} / \mathrm{ml})$ e 0 UI $\left(\circ_{-\circ}\right)$, 1UI $(\bullet \bullet)$ e $10 \mathrm{UI}(\bullet \bullet)$ de hCG. Média \pm EPM $(\mathrm{P}<0,01)$. 


\section{DISCUSSÃO}

A síntese de novo do colesterol é uma das maiores atividades biossintéticas das CG em folículos antrais (Gore-Langton e Armstrong, 1994) e ocorre, inicialmente, em resposta ao estímulo pelo FSH e, posteriormente, em resposta ao LH (Hsueh et al., 1984). Visto que o precursor da $\mathrm{P}_{4}$ é derivado da síntese de novo e também de estoques endógenos de lipídios (Simpson et al., 1980), torna-se possível a síntese de $\mathrm{P}_{4}$ pelas $C G$ cultivadas in vitro sem a adição suplementar de seu precursor.

$\mathrm{O}$ hCG estimulou o acúmulo de $\mathrm{P}_{4}$ pelas $\mathrm{CG}$ de folículo antral, presumivelmente pré-ovulatório, de égua na concentração de $1 \mathrm{UI}$ de $\mathrm{hCG} / \mathrm{ml}$ na presença de T. Estes resultados são semelhantes aos descritos por outros autores, que observaram aumento marcante na habilidade das CG em secretar $\mathrm{P}_{4}$ in vitro, na transição da fase folicular inicial para a fase folicular tardia (Sirois et al., 1991), em razão da exposição à concentração crescente de LH in vivo (Whitmore et al., 1973; Alexander e Irvine, 1982).

Alta concentração de hCG (10UI) não promoveu efeito estimulador na síntese de $\mathrm{P}_{4}$. A dessensibilização das células ovarianas às gonadotrofinas tem sido descrita em éguas (Jonassen e Richards, 1980; Jonassen et al., 1982) e em outras espécies como um mecanismo para prevenir a superestimulação das gônadas (Amsterdam et al., 2002). O processo de dessensibilização crônico é, em parte, atribuído ao acúmulo e internalização do complexo hormônio-receptor; o agudo pode ocorrer como resultado da fosforilação dos resíduos serina e treonina do receptor, impedindo que ele ative a formação do AMPc (Keren-Tal et al., 1996).

$\mathrm{O}$ hCG (1UI) estimulou o acúmulo de $\mathrm{P}_{4}$ no meio de cultura das $\mathrm{CG}$, porém esse aumento só foi observado na presença de testosterona. O resultado deste estudo inclui as CG de éguas no grupo das CG de outras espécies animais nas quais a regulação da síntese de $\mathrm{P}_{4}$ no folículo envolve a ação de andrógenos. Contudo, Tucker et al. (1991) observaram, em éguas, inefetividade da $\mathrm{T}$ em estimular a síntese/secreção de $\mathrm{P}_{4}$ in vitro, o que difere do efeito estimulador da $\mathrm{T}$ sobre o acúmulo de $\mathrm{P}_{4}$ em cultura de $\mathrm{CG}$ de eqüinos observado neste trabalho e por outros autores em suínos (Stoklosowa et al., 1982), bovinos (Fortune, 1986) e ratos (Lui e Hsueh, 1986). Em ratos, o efeito dos andrógenos é um fenômeno receptor mediado pelo LH (Liu e Hsueh, 1986) e adicionalmente estimulado pela presença de $\mathrm{E}_{2}$ (Hillier et al., 1977). Na produção de $\mathrm{P}_{4}$, a combinação da gonadotrofina com andrógenos atua não somente estimulando a síntese, mas também bloqueando a sua degradação (Moon et al., 1984).

O hCG não estimulou significativamente a secreção de $E_{2}$ pelas $C G$ acima das concentrações observadas em meio de cultura suplementado com $\mathrm{T}$ sem hCG, ou seja, não aumentou a atividade da P450aromatase (P450arom). Resultados semelhantes também foram observados por outros pesquisadores, ao utilizarem CG de éguas tratadas com FSH e/ou LH (Sirois et al., 1990; Tucker et al., 1991). Em CG de folículos pré-ovulatórios de bovinos in vivo foi demonstrado que o RNAm das enzimaschave da via sintética dos esteróides nas CG, como a P450 clivagem da cadeia lateral do colesterol (P450scc) e 3 $\beta$-hidroxi-esteróide dehidrogenase $/ \Delta^{5}-\Delta^{4}$-isomerase $\quad(3 \beta$-HSD), aumenta significativamente quando a fase folicular progride, porém esse aumento não ocorre com o RNAm da P450arom (Tian et al., 1995).

A T adicionada ao meio de cultura de CG de folículo antral presumivelmente pré-ovulatório de égua estimulou o acúmulo de $\mathrm{E}_{2}$ no meio de cultura, não apenas desempenhando papel de precursor do $E_{2}$, mas de potente estimulador da atividade da P450arom, pois a quantidade de $\mathrm{T}$ adicionada ao meio de cultura $(144 \mathrm{ng} / \mathrm{ml})$ foi bem menor do que a produção de $\mathrm{E}_{2}$ obtida com $6 \mathrm{~h}$ de cultura $(1119,74 \mathrm{ng} / \mathrm{ml})$, ou seja, 10,5 vezes maior. Sirois et al. (1991) observaram aumento na concentração de androstenediona no meio de cultura de $\mathrm{CG}$ de eqüinos durante três dias consecutivos. Contudo, quando não se adicionou $\mathrm{T}$ ao meio, a concentração de $\mathrm{E}_{2}$ foi muito baixa, alcançando $17 \mathrm{ng} / \mathrm{ml}$ às $144 \mathrm{~h}$ de cultura. Mais estudos são necessários para averiguar se esse 
andrógeno, utilizado como precursor para a síntese de $E_{2}$, seria oriundo da síntese pelas $C G$ ou liberação intracelular adicional. $\mathrm{O}$ efeito da $\mathrm{T}$ como precursor da síntese de $\mathrm{E}_{2}$ pelas $\mathrm{CG}$ foi relatado em eqüinos (Channing, 1969; Sirois et al., 1991) e em outras espécies animais (Dorrington et al., 1975; Erickson e Hsueh, 1978). Andrógenos também participam do mecanismo pelo qual o FSH aumenta a atividade da P450arom (Daniel e Armstrong, 1984). Porém, essa é a primeira vez que se demonstra em eqüinos o efeito da $\mathrm{T}$ como potente estimulador da síntese de $\mathrm{E}_{2}$, além de seu papel como precursor.

O fato do $\mathrm{LH} / \mathrm{hCG}$ não estimular a atividade da P450arom e a $\mathrm{T}$ apresentar efeito estimulador marcante na síntese de $\mathrm{E}_{2}$ sugere que, pelo menos durante a fase folicular tardia, a disponibilidade de substrato aromatizável seja limitante para que ocorra a síntese de $\mathrm{E}_{2}$ pelas CG. Essa hipótese é sustentada por relatos que demonstram que $\mathrm{CG}$ de folículos pré-ovulatórios de ovinos (Evans et al., 1981) e suínos (Tsang et al., 1985), cultivadas in vitro e coletadas durante diferentes tempos após o tratamento com gonadotrofina coriônica eqüina, apresentam atividade da P450arom constante. Tian et al. (1995) demonstraram, em bovinos, que a concentração do RNAm da P450arom se mantém sem alteração durante $24 \mathrm{~h}$ após a luteólise, e que o aumento marcante na síntese de $\mathrm{E}_{2}$, observado nesse período, foi devido ao aumento da concentração das enzimas-chave da via biossintética de andrógenos localizadas nas $\mathrm{CT}$

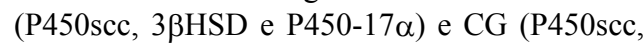
$3 \beta H S D)$, após elevação da freqüência de secreção do LH, aumentando a disponibilidade de substrato aromatizável. Outra evidência de que a disponibilidade de substrato aromatizável limita a síntese de $\mathrm{E}_{2}$ é que, após o pico préovulatório de $\mathrm{LH}$, a produção de $\mathrm{E}_{2}$ é inibida devido à diminuição da síntese de andrógenos pelas CT e células intersticiais (Lieberman et al., 1975; Katz e Armstrong, 1976).

Neste estudo foi observada relação temporal inversa entre concentrações de $\mathrm{E}_{2}$ e $\mathrm{P}_{4}$ no meio de cultura. Tem sido demonstrado que estrógenos diminuem a síntese/secreção de $\mathrm{P}_{4}$ pelas CG de suínos (Haney e Schomberg, 1978), bovinos (Fortune e Hansel, 1979), ratos (Hillier et al., 1977) e humanos (Bieszczad et al., 1982).
A ação inibitória do $E_{2}$ nas $C G$ de suínos é dependente do tempo de cultura e da concentração adicionada ao meio, mas não é dependente do grau de maturação folicular (Veldhuis, 1985). Estes dados sugerem que, após a diminuição das concentrações de $\mathrm{E}_{2}$ no meio de cultura, as CG se luteinizaram, aumentando a produção de $\mathrm{P}_{4}$ em resposta ao hCG e T, 24h após o início da cultura.

Os resultados permitem concluir que a testosterona modula a ação do LH na diferenciação e luteinização das células da granulosa e desempenha importante efeito estimulador na síntese/secreção do $\mathrm{E}_{2}$ pelas células da granulosa de folículo antral presumidamente pré-ovulatório de égua in vitro.

\section{AGRADECIMENTOS}

Os autores agradecem a Rogério Rosário de Azevedo, Roberta Vantini e Ivo Luiz de Almeida Júnior pela assistência técnica. Este projeto foi financiado pela FAPESP e CNPq.

\section{REFERÊNCIAS BIBLIOGRÁFICAS}

ALEXANDER, S.; IRVINE, C.H.G. Radioimmunoasay and in-vitro bioassay of serum LH throughout the equine oestrous cycle. J. Reprod. Fertil., v.32 (Suppl), p.253-260, 1982.

AMSTERDAM, A; HANOCH, T.; DANTES, A et al. Mechanisms of gonadotropin desensitization. Mol. Cell Endocrinol., v.187, p.69-74, 2002.

BÉlANGER, A.; CARON, S.; PICARD, V. Simultaneous radioimmunoassay of progestins, androgens and estrogens in rat testis. J. Steroid Biochem., v.13, p.185-190, 1980.

BIESZCZAD, R.R.; McCLINTOCK, J.S.; PEPE, G.J. et al. Progesterone secretion by granulosa cells from different sized follicles of human ovaries after short term incubation. $J$. Clin. Endocrinol. Metab., v.55, p.181-184, 1982.

CHANNING, C.P. Studies on tissue culture of equine ovarian cell types: Pathways of 
steroidogenesis. J. Endocrinol., v.43, p.403-413, 1969.

DANIEL, S.A.J.; ARMSTRONG, D.T. Site of action of androgens on follicle-stimulating hormone-induced aromatase activity in cultured rat granulosa cells. Endocrinology, v.114, p.1975-1982, 1984.

DORRINGTON, J.H.; MOON, Y.S.; ARMSTRONG, D.T. Estradiol-17 $\beta$ biosynthesis in cultured granulosa cells from hypophysectomized immature rats; stimulation by follicle stimulating hormone. Endocrinology, v.97, p.1328-1331, 1975.

ERICKSON, G.F.; HSUEH, A.J.W. Stimulation of aromatase activity by follicle-stimulating hormone in rat granulosa cells in vivo and in vitro. Endocrinology, v.102, p.1275-1282, 1978.

EVANS, G.; DOBIAS, M.; KING, G.L. et al. Estrogen, androgen, and progesterone biosynthesis by theca and granulosa of preovulatory follicles in the pig. Biol. Reprod., v.25, p.673-682, 1981.

FALK, B. Site of production of oestrogens in rat ovary as studied by microtransplants. Acta Physiol. Scand., v.47 (Suppl. 163), p.1-101, 1959.

FORTUNE, J.E. Bovine theca and granulosa cells interact to promote androgen production. Biol. Reprod., v.35, p.292-299, 1986.

FORTUNE, J.E.; HANSEL W. The effects of $17 \beta$-estradiol on progesterone secretion by bovine theca and granulosa cells. Endocrinology, v.104, p.1834-1838, 1979.

GOMES F.P. Curso de estatística experimental. Piracicaba: Livraria Nobel, 1987. 467p.

GORE-LENGTON, R.E.; ARMSTRONG, D.T. Follicular steroidogenesis and its control. In: KNOBIL, E.; NEIL, J.D. (Eds.). The Physiology of reproduction. 2.ed. New York: Raven, 1994. V.1, p.571-627.

HANEY, AF.; SCHOMBERG, D.W. Steroidal modulation of progesterone secretion by granulosa cells from large porcine follicles: a role for androgens and estrogens in controlling steroidogenesis. Biol. Reprod., v.19, p.242-248, 1978.
HILLIER, S.G.; KNAZEK, R.A.; ROSS, E.T. Androgenic stimulation of progesterone production by granulosa cells from preantral ovarian follicles: further in vitro studies using replicate cell cultures. Endocrinology, v.100, p.1539-1549, 1977.

HSUEH, A.J.W.; ADASHI, E.Y.P.; JONES, P.C.B. et al. Hormonal regulation of the differentiation of cultured ovarian granulosa cells. Endocr. Rev., v.5, p.76-127, 1984.

JONASSEN, J.A.; BOSE, K.; RICHARDS, J.S. Enhancement and desensitization of hormoneresponsive adenylate cyclase in granulosa cells of preantral and antral ovarian follicles: effects of estradiol and follicle-stimulating hormone. Endocrinology, v.111, p.74-79, 1982.

JONASSEN, J.A.; RICHARDS, J.S. Granulosa cell desensitization: effects of gonadotropins on antral and preantral follicles. Endocrinology, v.106, p.1786-1794, 1980.

KATZ, Y.; ARMSTRONG, D.T. Inhibition of ovarian estradiol-17 $\beta$ secretion by luteinizing hormone in prepubertal, pregnant mare serumtreated rats. Endocrinology, v.99, p.1442-1447, 1976.

KELLY, C.M.; HOYER, P.B.; WISE, M.E. Invitro and in-vivo responsiveness of the corpus luteum of the mare to gonadotrophin stimulation. J. Reprod. Fertil.,v.84, p.593-600, 1988.

KEREN-TAL, I.; DANTES, A.; AMSTERDAN, A. Activation of FSH-responsive adenylate cyclase by staurosporine: Role for protein phosphorylation in gonadotropin receptor desensitization. Mol. Cell. Endocrinol., v.116, p.39-48, 1996.

LIEBERMAN, M.E.; BARNEA, A.; BAUMINGER, S. et al. LH effect on the pattern of steroidogenesis in cultured Graafian follicles of the rat: dependence on macromolecular synthesis. Endocrinology, v.96, p.1533-1542, 1975.

LIU, Y.; HSUEH, A.J.W. Synergism between granulosa and theca-intersticial cells in estrogen biosynthesis by gonadotrophin-treated rat ovaries: Studies on the two-cell, twogonadotrofin hypothesis using steroid antisera. Biol. Reprod., v.35, p.27-36, 1986. 
MOON, Y.S.; DULEBA, A.J.; TAKAHASHI, H. Differential actions of FSH and androgens on progesterone catabolism by rat granulosa cells. Bioch. Bioph. Res. Commun., v.199, p.694-699, 1984.

ROSA E SILVA, A.A.M.; CALDAS, M.C.S.; CAMPOS, L.M. et al. Setting-up and validation of two radioimmunoassay methods for determination of plasma progesterone concentration in mares, cows and rats. Ars Vet., v.9, p.1-9, 1993.

SIMPSON, E.R.; ROCHELLE, D.B.; CARR, B.R. et al. Plasma lipoproteins in follicular fluid of human ovaries. J. Clin. Endocrinol. Metab., v.51, p.1469-1471, 1980.

SIROIS, J.; KIMMICH, T.L.; FORTUNE, J.E. Developmental changes in steroidogenesis by equine preovulatory follicles: effects of equine luteinizing hormone, follicle-stimulating hormone, and chorionic gonadotropin. Endocrinology, v.127, p.2424-2430, 1990.

SIROIS, J.; KIMMICH, T.L.; FORTUNE, J.E. Steroidogenesis by equine preovulatory follicles: relative roles of theca interna and granulosa cells. Endocrinology, v.128, p.1159-1166, 1991.

STOKLOSOWA, S.; GREGORASZCUK, E.; CHANNING, C.P. Estrogen and progesterone secretion by isolated cultured porcine thecal and granulosa cells. Biol. Reprod., v.26, p.943-952, 1982.
TIAN, X.C.; BERNDTSON, A.K.; FORTUNE, J.E. Differentiation of bovine preovulatory follicles during the follicular phase is associated with increases in messenger ribonucleic acid for cytochrome $\mathrm{P} 450$ side-chain cleavage, $3 \beta$ hydroxysteroid dehydrogenase, and P450 17 $\alpha$ hidroxylase, but not P450 aromatase. Endocrinology, v.136, p.5102-5110, 1995.

TSANG, B.K.; AINSWORTH, L.; DOWNEY, B.R. et al. Differential production of steroids by dispersed granulosa and theca interna cells from developing preovulatory follicles of pigs. $J$. Reprod. Fertil., v.74, p.459-471, 1985.

TUCKER, K.E.; HENDERSON, K.A.; DUBY, R.T. In vitro steroidogenesis by granulosa cells from equine pre-ovulatory follicles. J. Reprod. Fertil., v.44 (Suppl), p.45-55, 1991.

VELDHUIS, J.D. Bipotential actions of estrogens on progesterone biosynthesis by ovarian cells. II. Relation of estradiol's stimulatory actions to cholesterol and progestin metabolism in cultured swine granulosa cells. Endocrinology, v.117, p.1076-1083, 1985.

WHITMORE, H.L.; WENTWORTH, B.C.; GINTHER, O.J. Circulating concentrations of luteinizing hormone during estrous cycle of mares as determined by radioimmunoassay. Am. J. Vet. Res., v.34, p.631-636, 1973. 